\title{
Convenciones y disrupciones en la cobertura de los eventos políticos en la TV lineal y social. Análisis de la noche de las elecciones del $26 \mathrm{M}$ de 2019
}

\section{Conventions and disruptions in the coverage of political events in linear and social TV. 2019 26M election night analysis}

\author{
Ana-Isabel Rodríguez-Vázquez; Alba Silva-Rodríguez; Sabela Direito-Rebollal; Berta \\ García-Orosa
}

Cómo citar este artículo:

Rodríguez-Vázquez, Ana-Isabel; Silva-Rodríguez, Alba; Direito-Rebollal, Sabela; García-Orosa, Berta (2020). "Convenciones y disrupciones en la cobertura de los eventos políticos en la TV lineal y social". El profesional de la información, v. 29, n. 2, e290205.

https://doi.org/10.3145/epi.2020.mar.05

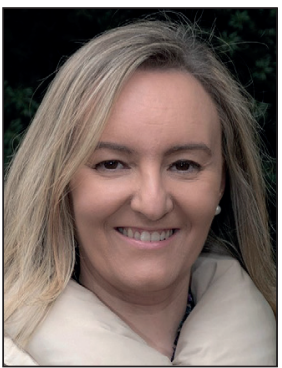

Ana-Isabel Rodríguez-Vázquez https://orcid.org/0000-0001-7975-1402 Universidade de Santiago de Compostela Facultad de Ciencias de la Comunicación Av. Castelao, s/n. 15782 Santiago de Compostela, España anaisabel.rodriguez.vazquez@usc.es

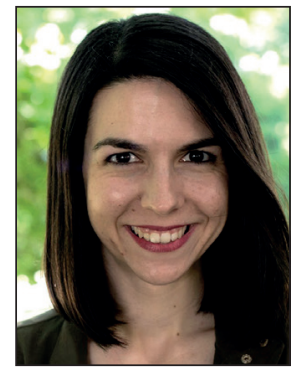

Sabela Direito-Rebollal https://orcid.org/0000-0002-0189-4451

Universidade de Santiago de Compostela Facultad de Ciencias de la Comunicación Av. Castelao, s/n.

15782 Santiago de Compostela, España sabela.direito@usc.es

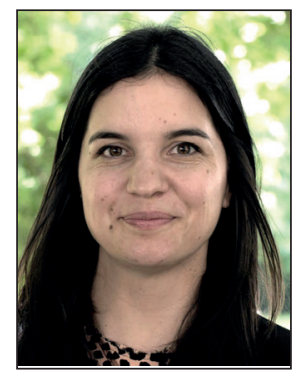

Alba Silva-Rodríguez

https://orcid.org/0000-0002-1221-5178

Universidade de Santiago de Compostela Facultad de Ciencias de la Comunicación Av. Castelao, s/n. 15782 Santiago de Compostela, España alba.silva@usc.es

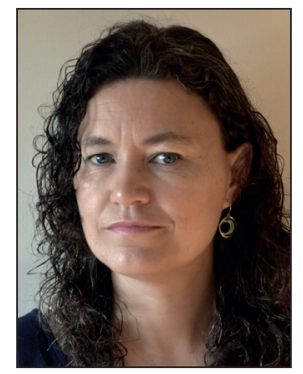

Berta García-Orosa $\triangle$ https://orcid.org/0000-0001-6126-7401

Universidade de Santiago de Compostela Facultad de Ciencias de la Comunicación Av. Castelao, $\mathrm{s} / \mathrm{n}$.

15782 Santiago de Compostela, España berta.garcia@usc.es

\section{Resumen}

Mediante un método mixto se analiza la oferta lineal (en directo), social (redes sociales) y de participación de la audiencia (live-tweeting) durante el especial informativo de la noche electoral del 26 de mayo de 2019 (comicios europeos, autonómicos y municipales) en La 1 de TVE y La sexta. Se parte de la hipótesis de que el consumo de televisión ha cambiado con la competencia de las plataformas de visionado a la carta. El objetivo de este trabajo empírico es determinar la estrategia informativa multicanal llevada a cabo por las citadas emisoras y conocer la respuesta de la audiencia. Eventos

Financiación

Este artículo se ha elaborado en el marco del proyecto Cibermedios nativos digitales en España: formatos narrativos y estrategia móvil (RTI2018-093346-B-C33), del Ministerio de Ciencia, Innovación y Universidades, cofinanciado por el Fondo Europeo de Desarrollo Regional (Feder).

La autora Sabela Direito-Rebollal es beneficiaria del programa para la Formación del Profesorado Universitario del Ministerio de Universidades (Gobierno de España). 
políticos como los procesos electorales mantienen a la audiencia conectada con la televisión tradicional y la social, y generan altos volúmenes de conversación.

\title{
Palabras clave
}

Televisión lineal; Televisión social; Redes sociales; Medios sociales; Programación; Audiencias; Eventos políticos en TV; Información electoral; Elecciones; Comunicación política.

\begin{abstract}
Through a mixed method, the linear (live), social (social media) and audience participation (live-tweeting) offer during the special information program of March, 26th 2019 electoral night in La 1 of TVE and La sexta is analysed. We had the hypothesis that television consumption has changed with the competition of VoD-OTT platforms. The objective of this empirical work is to determine the multichannel information strategy carried out by the mentioned stations, and to know the response of the audience. Political events such as electoral processes keep the audience connected to traditional and social television, and generate high volumes of conversation.
\end{abstract}

\section{Keywords}

Linear television; Social television; Social networks; Social media; Programming; Audiences; Political events on TV; Electoral information; Elections; Political communication.

\section{Introducción y contexto}

El actual modelo de difusión televisiva, que combina una cobertura lineal (TDT-parrilla clásica de programación 24 horas al día, 365 días al año), en diferido (plataformas Over-The-Top conocidas como OTT-VoD) y social (televisión comentada en redes sociales), obliga a los canales a diseñar una oferta múltiple y combinada. Aunque las OTT se consolidan, datos del mercado estadounidense (Comscore, 2019) reflejan que el consumo de la TV lineal sigue siendo muy elevado y conversacionalmente activo. Las audiencias surfean con gran facilidad en la era del consumo multipantalla y comentan en tiempo real los contenidos que les resultan atractivos, especialmente realities y eventos de actualidad, tanto a través de redes sociales como de grupos privados de mensajería.

La participación de la audiencia se percibe no únicamente como activa en la interpretación de los mensajes periodísticos, sino también en la producción y el debate de contenidos (Masip; Ruiz-Caballero; Suau, 2019). Se refuerza el concepto de televisión social cuando canales alternativos como las redes sociales sirven de soporte conversacional en torno a lo que sucede en una programación (Gallego, 2002). Los receptores utilizan las plataformas para comentar y, a veces, influir en la emisión en directo en la televisión. El consumo lineal combina la actitud pasiva del espectador (Saavedra-Llamas; Rodríguez-Fernández; Barón-Dulce, 2015) con el activismo que surge del proceso de fragmentación de la audiencia tradicional (Masip; Ruiz-Caballero; Suau, 2019), en un ecosistema de medios dominado por los móviles, internet y las redes sociales. La búsqueda del compromiso con este espectador en ambos modelos televisivos (lineal y social) forma parte de las estrategias habituales de los canales, pero cobra especial relevancia cuando aumentan las expectativas de audiencia en torno a una emisión televisiva por el interés que suscitan determinados contenidos, como es el caso analizado en este artículo.

Frente a las amenazas de los cambios dinámicos en el mercado del vídeo y la televisión tradicional, el uso frecuente de redes sociales podría ser una oportunidad para aumentar el visionado de televisión. Desde 2010, las transmisiones de grandes eventos como los Juegos Olímpicos, la Super Bowl y los Grammys, obtuvieron resultados de audiencia más altos en Estados Unidos con la incorporación de las redes al modelo de consumo televisivo (Cha, 2016). También desde entonces, en España los medios sociales reforzaron las emisiones televisivas de eventos como el festival de Eurovisión, realities como Operación triunfo, Gran hermano y Supervivientes, y eventos políticos como el debate entre los candidatos a la Presidencia del Gobierno del 24 de abril de 2019, o la cobertura de la noche electoral el 28 de abril de ese mismo año.

Livingstone y Lunt (1994) ya analizaban a mediados de la década de 1990 los efectos de la participación activa del público en televisión, y se preguntaban cómo se gestionaba esa relación en los programas de discusión abiertos a la participación ciudadana. Más de veinte años después, el valor y la importancia de la discusión pública y el debate en televisión se redefinen en un escenario dominado por las redes sociales. La facilidad de uso de plataformas como Twitter y su consolidada popularidad la han convertido en un foro para el debate y la participación pública (Burnett; Bloice, 2016). La red de micro-blogging se consolida como un importante backchannel para los mass media (Harrington; Highfield; Bruns, 2013), mediante el cual interactúan con una audiencia que se muestra especialmente activa a la hora de comentar los debates políticos (Bruns; Burgess 2011; Shamma; Kennedy; Churchill, 2009).

En este escenario, la comunicación política en televisión ha evolucionado hacia un sistema híbrido (Chadwick, 2013; Shah et al., 2015; García-Orosa; López-García, 2018) en el que los principales actores (partidos, medios y audiencias) interactúan a través de diferentes plataformas. Los mediated events que se producen en una campaña electoral son uno de los momentos con mayor participación y relevancia (Jungherr, 2016). Spyridou (2019) señala cómo el sentimiento de eficacia política y los más jóvenes de la sociedad pueden provocar una mayor participación, confirmando las conexiones existentes entre el empoderamiento de los ciudadanos, el compromiso político y lo que se llamó periodismo participati- 
vo. Además, los mensajes políticos se incrementan en estos eventos especiales mediados (Jürgens; Jungherr, 2015; Lin et al., 2014) con una intensificación de la participación en determinados momentos del evento (Jungherr, 2016).

La comunicación política camina también hacia una comunicación híbrida en la que las redes sociales y, sobre todo Twitter, adquieren una presencia importante en todas las campañas electorales con los partidos políticos intentando acceder a la opinión pública y buscando el engagement con el ciudadano. La comunicación bidireccional en tiempo real, la diseminación de la información, la autopromoción y el marketing e, incluso la capacidad de las redes sociales como modelo de predicción (Grčić; Babac; Podobnik, 2017), las convirtieron en un elemento esencial en las campañas electorales. En la bibliografía existente (Utz; Schultz; Glocka, 2013; Vesnic-Alujevic, 2012; Abitbol; Lee, 2017; Ji et al., 2018; Gálvez-Rodríguez; Caba-Pérez; López-Godoy, 2014; Painter, 2015) se destaca la bidireccionalidad, la interactividad, las oportunidades de diálogo e incluso la capacidad para provocar una democracia participativa y deliberativa. Sin embargo, algunos investigadores comentan el riesgo que supone el uso de las redes sociales en crisis (Ott; Theunissen, 2015) y se cuestiona la relación entre lo que los académicos observan como potencial y la efectividad de los medios sociales (Taylor; Kent, 2010).

Entre las redes sociales merece una mención especial Twitter, que es la más analizada en el ámbito de la comunicación organizacional política (Dodd; Collins, 2017; Gálvez-Rodríguez et al., 2018). Su uso es percibido positivamente ya que aumenta la credibilidad, la conversación, la comunicación afectiva y mitiga la culpa en situaciones de crisis (Liu; Lai; Xu, 2018). Los investigadores resaltan el potencial interactivo (Waters; Jamal, 2011) y la mayor actividad en esta plataforma se registra en campaña electoral (cuando los partidos y candidatos se sirven de Twitter para proporcionar información o enlazar a su web) y, sobre todo, en el caso de los media events (Jungherr, 2016). Los usuarios tienden a ofrecer contexto fáctico a los eventos en pantalla, por ejemplo, al vincularse a otro contenido en la Web, a menudo refutando las declaraciones y posiciones expuestas por los políticos destacados en los eventos (Jungherr, 2016). Un sentido de comunidad, vinculación social con las redes existentes, refuerzo de una persona online, entretenimiento, intercambio de información, movimiento social, autodocumentación e incentivos forman parte de las motivaciones de los usuarios que fortalecen esta retroalimentación (Alonso-González, 2018; Azurmendi, 2018; Cha, 2016). También hay indicios de que, durante los eventos mediados, la atención de los usuarios de Twitter se concentra en un número menor de hashtags y actores, y de que los políticos se convierten en el centro de atención (Jungherr, 2016).

Este artículo propone el análisis de un evento político (political mediated event) especialmente relevante por el contexto de activación política de la sociedad española y por la posibilidad de comparar el objeto de estudio en diferentes ámbitos electorales: comicios europeos, autonómicos y municipales. Durante la programación televisiva de la noche electoral del 26 de mayo de 2019 confluyeron estrategias de los tres actores implicados en un repertorio de canales de comunicación. La cobertura "en vivo" combinó la oferta en TV lineal y redes sociales con una estrategia para sumar la atención del público en un doble escenario:

- el del espectador del programa "clásico" de televisión, que se contabiliza con el dato de audimetría;

- el de la audiencia más activa interesada en participar y comentar, que se contempla con el dato de audiencia social.

\section{Objetivo y metodología}

El objetivo fundamental de este trabajo es analizar las coberturas especiales informativas de las cadenas televisivas españolas, en concreto de La 1 y La sexta, en el doble modelo televisivo. El fin es observar la oferta de contenidos de la emisión online y sus publicaciones en redes sociales, para determinar la estrategia informativa multicanal y ofrecer un resultado combinado con la respuesta de la audiencia. El contexto elegido para el estudio son las elecciones del 26 de mayo de 2019. Estos comicios adquirieron especial relevancia para la investigación por varios motivos:

- la alta activación política de la población;

- la confluencia de diferentes procesos electorales;

- unos medios de comunicación muy implicados en las campañas electorales.

Se planteó, pues, un trabajo empírico sobre una realidad determinada que se tradujo en una investigación teórico-observacional basada en un método mixto (cuantitativo y cualitativo). El estudio parte de una observación coincidental de la oferta lineal (emisión en directo), social (publicaciones en RRSS) y de participación de la audiencia (live-tweeting) durante la cobertura especial informativa de la noche electoral del 26M de 2019, desde las 19:25h (en el caso de La sexta) y las 19:53h (en La 1 de TVE), hasta las 02:14h y 02:05h en que terminaron las emisiones de ambos programas.

Partiendo de la hipótesis de que la televisión lineal sigue siendo un gran escenario para la comunicación política en momentos electorales gracias, entre otros formatos, a los debates y los especiales de las noches electorales, optamos por una muestra de conveniencia y tomamos la decisión de apostar por estos dos canales basándonos en datos previos:

- como cadena pública con gran tradición informativa electoral, La 1 ostenta también el récord del debate electoral más visto de la última década (Mariano Rajoy vs. José Luis Rodríguez Zapatero el 25 de febrero de 2008, con una audiencia media de 8.017.000 espectadores y un 36,4\% de share, según informes de Barlovento Comunicación con datos de Kantar Media);

- La sexta se ha consolidado como el canal con mayor seguimiento de la audiencia en las citas electorales previas. EI dato más reciente de que disponíamos se correspondía con la cobertura de la noche electoral de las Generales del 28A donde Al rojo vivo: Edición especial objetivo La Moncloa fue líder entre las 21:14h y las 23:47h con 4.044 .000 de espectadores y un $20,7 \%$ de share. 
Con ello se pretendió cubrir los siguientes objetivos:

O1) Analizar las coberturas especiales informativas de La 1 y La sexta durante la noche electoral del 26M en su emisión lineal (escaletas de los programas para ver contenidos como los temas, focos informativos, protagonistas de las tertulias de análisis...) y en el escenario social (oferta de ambas cadenas en Twitter, Facebook, Instagram y YouTube, para contrastar las estrategias de ambos canales en sus ofertas informativas a través de líneas de tiempo comparativas de contenidos online -con confrontación de escaletas-y en social -frecuencia de publicación, elementos narrativos (texto, imagen fija, vídeo, audio, infografía, etc.)-.

O2) Analizar el engagement de la audiencia en torno a este evento informativo, valorando el dato de audimetría y la interacción en las redes sociales. Teniendo en cuenta que en los medios sociales digitales los usuarios forman redes cuando interactúan con otros, el estudio de estas conexiones permite dilucidar hasta qué punto están los usuarios interconectados entre sí y cómo fluye la información entre ellos (Himelboim et al., 2017). En este caso, el análisis se ha focalizado en Twitter por ser la red que tiene una mayor capacidad para favorecer la interacción entre ciudadanos, periodistas y políticos (López-Rabadán; Mellado, 2019). Así mismo, es también la plataforma sobre la que las cadenas de televisión centraron su actividad durante la emisión de los especiales electorales del 26 de mayo.

Para recoger los datos relacionados con el $\mathrm{O} 2$ se ha utilizado el software NodeXL Pro, que permite extraer un máximo de 18.000 tweets recuperados en base a una palabra clave, hashtag o nombre de usuario. A fin de obtener el mayor número posible de tweets, la captura se ha realizado en tiempo real, ejecutando una búsqueda cada hora desde el inicio de la emisión de ambos programas. Con respecto a la query, la consulta se ha efectuado en base al hashtag oficial de los formatos objeto de estudio: \#SuperDomingoL6 (La sexta) y \#26MTúDecides (La 1). El dataset final ha quedado conformado por un total de 26.753 tweets (incluyendo retweets, menciones y respuestas) para Al rojo vivo: edición especial. Objetivo superdomingo y de 4.680 para el 26M: Tú decides. Posteriormente, los datos se importaron en Gephi con el fin de visualizar las relaciones entre los usuarios que comentaron en directo ambos especiales. Siguiendo el modelo de investigaciones anteriores (Hawthorne; Houston; McKinney, 2013; Larsson; Moe, 2012), se optó por generar un grafo para representar la red de retweets y otro para las menciones. En los dos casos se ha empleado el grado de entrada como medida que refleja la receptividad y popularidad que un actor tiene en la red (Wasserman; Faust, 2013). Los usuarios con conexiones en común se han agrupado a través del algoritmo de modularidad de Blondel et al. (2008) disponible en Gephi. El tamaño de los nodos es proporcional al número de veces que han sido retweeteados o mencionados, mientras que su color indica la pertenencia a una determinada comunidad.

La investigación se completó con dos entrevistas en profundidad a Estefanía de Antonio, redactora jefa en RTVE.es en Radiotelevisión Española y a Ángela Bonachera, responsable de la gestión de redes sociales de La sexta.

\section{Análisis y resultados}

\subsection{Cobertura informativa de la noche electoral del 26M: Programación lineal y gestión de RRSS}

\subsubsection{Programación: formatos especiales en la emisión lineal}

La cobertura especial informativa durante la noche electoral del 26M en La 1 de TVE y La sexta tuvo una duración similar (unas siete horas) entre las 19:25h (poco antes del cierre de los colegios electorales) y las 02:14h (con el escrutinio en niveles del 90\%), pero el primero en posicionarse ante la audiencia fue Al rojo vivo: edición especial. Objetivo superdomingo (La sexta), unos 30 minutos antes que su rival 26M: Tú decides (La 1). Ambos canales desplegaron una oferta lineal con escaletas muy similares estructuradas en cinco bloques:

1) Apertura con la ronda de conexiones con los reporteros desplegados en las sedes de los partidos ( $P P, P S O E, C i u d a d a-$ nos, Unidas Podemos y Vox). A continuación, resumen de lo que había sido la jornada electoral recogiendo las votaciones de los principales candidatos, incidencias en las urnas y datos de participación de las 18:00h. Todo ello alternado con los comentarios de los tertulianos en el plató: José Miguel Contreras, José Antonio Zarzalejos, María Claver, Lluís Orriols, Ignacio Escolar, Angélica Rubio y Jesús Cintora, en La sexta; y Mayte Alcaraz, Arsenio Escolar, Alejandro Requeijo, Francisco Camas, Lucía Méndez, Manuel Mostaza, Esther Palomera y Argelia Queralt, en La 1.

2) Avance de resultados de las encuestas a pie de urna, especialmente centrados en las municipales y autonómicas. Se adelanta La sexta con la primera encuesta sobre el Ayuntamiento de Barcelona a las 20:01h para centrarse a las 20:04h en Madrid -Comunidad y Ayuntamiento-. La 1 ofrece información sobre los sondeos a partir de las 20:10h.

3) Primeros resultados con voto escrutado. De nuevo es $L a$ sexta la que adelanta a las 21:46h los resultados en Asturias, Aragón, Sevilla, Bilbao, Cádiz, San Sebastián, Madrid, Barcelona, Murcia, Málaga, Badalona, Valencia, Castilla-La Mancha, Fuenlabrada, Burgos y Extremadura, hasta las 22:34h. Los primeros datos procedentes del escrutinio que ofrece $L a$ 1 son los de El Ejido (Almería), a las 22:04h, y los de Madrid llegan alrededor de las 22:34h a través de la colaboradora Esther Palomera, del Huffington Post.

4) Alerta sobre el vuelco de las encuestas en Madrid. Se produce un punto de inflexión en ambos programas, como se verá también en las redes sociales: sobre las 22:47h Ferreras adelanta que los gobiernos de Madrid "están en el aire" y ambos canales confirman alrededor de las 23:15h, con el 55\% de voto escrutado, que Manuela Carmena ya puede perder la alcaldía (los datos de la Comunidad de Madrid son posteriores). Otro ayuntamiento con gran protagonismo en la 
cobertura informativa de la noche electoral es Barcelona y, de nuevo, es La sexta quien adelanta los resultados (23:50h) hasta que ambas coinciden en la comparecencia de Ada Colau y otros líderes autonómicos a partir de la medianoche.

5) Repaso por los resultados de distintas localidades y despedida: coincidencias en focos informativos como Castilla y León, Castilla-La Mancha, Cádiz, Galicia, Cantabria, Aragón, Extremadura, Canarias o Ceuta y Melilla.

Aunque la estructura de las escaletas fue similar, se observaron diferencias notables entre ambos formatos. Por ejemplo, el enfoque de apertura de La sexta fue directo, con conexiones en las sedes de los partidos y un pulso continuado sobre los datos y la actualidad más urgente. La 1 apostó por una apertura más sosegada en el fondo y en la forma, explicativa del formato, con presentación de elementos diferenciadores como el público o los efectos visuales incorporados en la escenografía, y con un enfoque de sus conexiones iniciales centradas en las localidades donde los pactos podrían tener la clave de la gobernabilidad.

La sexta marca desde un primer momento dos focos clave en su cobertura informativa (Madrid y Barcelona), que poco a poco abren a lo largo del especial informativo, mientras que $L a 1$ ya mantiene un foco múltiple desde el inicio del programa y ofrece una mayor variedad de contenidos. El esquema de La sexta es más reducido, muy cíclico, con un único presentador principal (Antonio García Ferreras), con conexiones muy localizadas y gran protagonismo de la tertulia del plató. La oferta de La 1, por el contrario, se amplifica con reportajes variados como una visita por los despachos de las alcaldías de las ciudades importantes de España, un recorrido por municipios pequeños como Castejón de Henares o por sedes de periódicos provinciales, entrevistas con el rapero Arkano sobre el problema de las fake-news, o con expertos sobre la Unión Europea como Carlos Westendorpp o José María Gil-Robles que conducen dos presentadores principales (Ana Blanco y Carlos Franganillo). También destaca el despliegue de La 1 en la cobertura de las elecciones europeas con conexiones en directo con el centro de datos en Bruselas y capitales como París, Berlín, Londres, Roma y Lisboa a través de su red de corresponsales. Sin embargo, La sexta sólo dispuso de una fuente informativa en la sede del Parlamento Europeo. Con todo, la agilidad del ritmo narrativo de La sexta parece lograr un mayor engagement en la audiencia lineal, según los datos de audimetría (imagen 1).

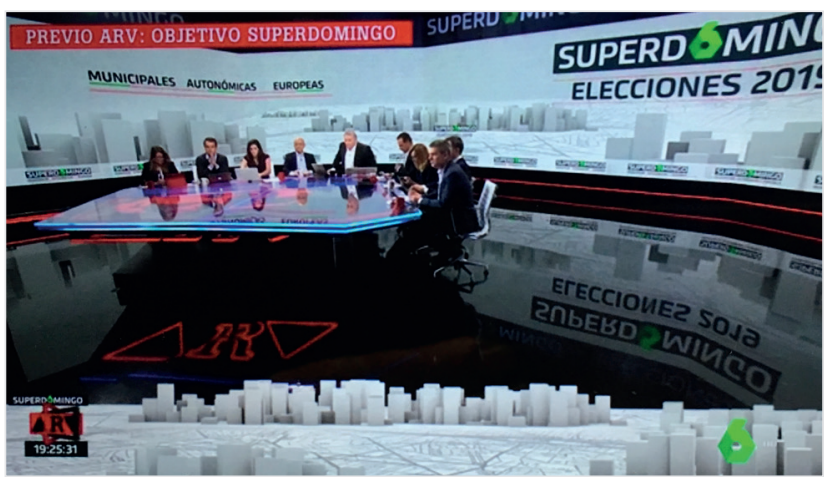

Al rojo vivo: edición especial. Objetivo superdomingo Previo (29:25h - 21:27h): 1.551 .000 (12,1\% share)

Programa (21:27h - 23:33h): $2.573 .000(14,3 \%)$

Post $(23: 33 \mathrm{~h}-02: 14 \mathrm{~h}): 1.092 .000(11,5 \%)$

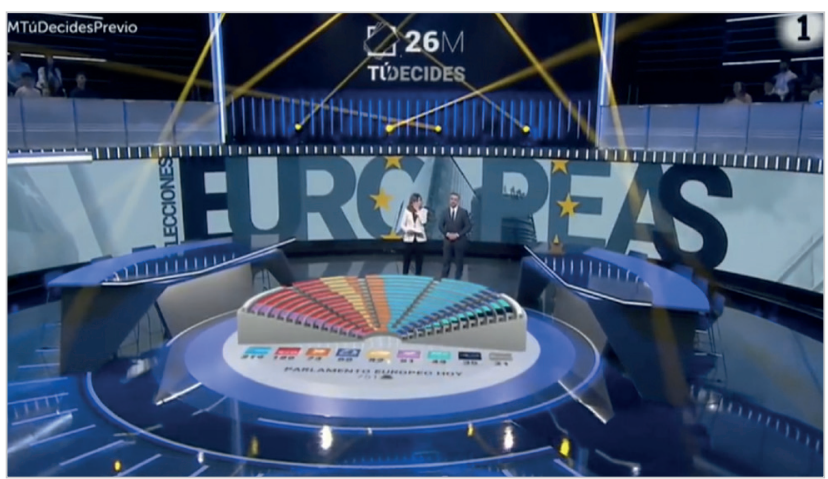

26M: Tú decides

Previo (19:53h - 21:06h): 882.000 (7\%)

Programa (21:06h - 02:05h): $1.015 .000(7,4 \%)$

Imagen 1. Audiencias lineales de los especiales informativos del 26M.

Elaborado con datos de Kantar Media

El análisis de la cobertura televisiva en la noche electoral del 26M se completó con la observación de las plataformas sociales, entendiendo que es el método más adecuado para ver las relaciones que se producen entre los actores de una red (Haythornthwaite, 1996):

- en Twitter se analizaron las publicaciones promovidas por cada uno de los dos canales con los hashtags \#SuperDomingoL6 y \#26MTúDecides y a través de sus diferentes perfiles (tres en el caso de La sexta: @DebatAlRojoVivo, @sextaNoticias, @laSextaTV; y otros cinco en La 1: @24h_tve, @telediario_tve, @La1_tve, @rtve, @tve. Se excluyeron las cuentas de los centros territoriales de TVE);

- en Facebook, La sexta optó por volcar su cobertura en los perfiles de sus programas informativos Al rojo vivo y La sexta noticias, en la cuenta del canal La sexta y, más residualmente, en la corporativa Atresplayer. Por el contrario, la cobertura de la noche electoral en TVE se concentró en tres perfiles: el canal informativo $24 h$, la generalista TVE y la corporativa RTVE (no se localizaron cuentas de los telediarios ni de La 1 en esta red social).

Tanto la cadena pública como la privada disponen de un plan de difusión de contenidos o guía de uso para redes sociales. Dentro de la estrategia de RTVE se contempla la segmentación de publicaciones de acuerdo con el perfil de usuario al que van dirigidas. Esto no sucede en los perfiles de La sexta porque, según la responsable de redes,

"entendemos que somos un medio generalista y tenemos que llegar a la mayor parte de gente posible".

A la hora de seleccionar los hashtags para la red social Twitter las dos entrevistadas coinciden en que la estrategia suele 
ser pragmática. Ángela Bonachera afirma que siempre intentan incluir la marca de la cadena y que se guían por los dictámenes de la emisión lineal.

"Era un superdomingo, elecciones europeas, municipales... esa era la idea que la cadena quería usar".

Estefanía de Antonio explica que apostaron por emplear el nombre del programa especial de TVE durante toda la jornada a través del hashtag \#26MTúDecides.

En esta fase del trabajo se identificaron todos los posts que fueron publicados desde el canal, con el objetivo de determinar el esfuerzo realizado por La 1 y La sexta en la cobertura de la noche electoral en sus RRSS, analizando aspectos como la temática de las publicaciones (autonómica, municipal o europea), los principales focos informativos, la frecuencia de publicación, los recursos empleados (vídeo, fotografía, infografía...) y el nivel de interacción básica con los usuarios a través de comentarios y retweets para identificar estrategias. Se aplicó este análisis combinado (de contenido y cuantitativo) sobre un total de 852 posts que conformaron el universo total (100\%) de publicaciones generadas por ambos medios. De esta exploración inicial ya se deduce el mayor peso estratégico que las televisiones otorgan a Twitter durante esta cobertura informativa especial, ya que sólo 69 de los 440 posts de La sexta se localizaron en Facebook, mientras que TVE únicamente realizó 39 publicaciones en Facebook, frente a las 374 de Twitter.

Además de la escasez de información (en términos cuantitativos) y de la menor frecuencia de publicación que se registra en Facebook, no se ha identificado una estrategia de diseño específica para cada red social. Sin embargo, las profesionales entrevistadas afirman que sí que se produce contenido adaptado a cada plataforma. Ángela Bonachera considera que

"no es igual el lenguaje de Twitter que el de Facebook o Instagram".

Para tener una perspectiva completa, ampliamos el análisis de las RRSS al seguimiento de las publicaciones realizadas por La sexta y La 1 en los perfiles de Instagram y en los canales de YouTube. Se detecta una notable diferencia entre ambas ofertas. La 1 trabajó con dos perfiles en Instagram: en 24horas el esfuerzo de cobertura informativa se centró en la elaboración de stories exclusivas para la audiencia de esta red social, con un seguimiento de las primeras horas de la jornada electoral, reservando para la cuenta de RTVE las stories sobre los resultados electorales que se publicaron simultáneamente a la emisión en lineal.

Así lo confirma la redactora jefa de RTVE.es, quien apunta que

"se decidió que el canal de $24 \mathrm{~h}$ de Instagram diera titulares generales y dejar el perfil de RTVE para aportar más detalles en esta red social".

Estefanía de Antonio afirma que se reservó el live clipping para Twitter. En La sexta Ángela Bonachera explica que se publicó en Instagram con una frecuencia mínima de cinco minutos anunciando en directo los resultados de las elecciones.

En YouTube, TVE ofreció en simulcast la señal de su emisión lineal y, además, creó un repositorio específico para compilar los vídeos sobre el $26 \mathrm{M}$ que permanece accesible al usuario. Por el contrario, la visibilidad de La sexta en YouTube fue nula.

Dado que se evidencia un mayor esfuerzo de los canales en torno a Twitter por la facilidad de uso, la participación en tiempo real y la interacción con otros usuarios y con el propio programa (Orihuela, 2011), la segunda fase (análisis de contenido) y la tercera (análisis de la conversación) se centran en esta plataforma. Ángela Bonachera y Estefanía de Antonio coinciden al destacar las fortalezas de Twitter para la difusión del especial de la noche electoral del 26M. La responsable de medios sociales de La sexta indica que Twitter permite emitir en vivo y ofrecer información actualizada e instantánea. Desde La sexta se decantan por publicar en la red de micro-blogging utilizando, preferentemente, texto e imágenes.

Para el análisis se tomaron como referencia los perfiles del canal que fueron apareciendo bajo los hashtags oficiales promocionados en las emisiones de la TV lineal, como se explicó al inicio de este epígrafe. La estrategia es muy distinta y una de las principales diferencias que se observan en el diseño de las publicaciones es el uso del vídeo. La sexta sólo publicó dos vídeos, coincidiendo con el inicio de las emisiones en lineal:

- a las 19:29h publicó un vídeo promocional del arranque del programa especial, que logró 46,4 mil reproducciones;

- a las 19:49h el segundo y último vídeo recordando el paso por los platós de la cadena de los candidatos europeos.

Se identifica una posible justificación: la estructura de los equipos que gestionan las redes sociales en los dos medios objeto de estudio también es muy diferente. Mientras que en RTVE existe un departamento formado por cuatro profesionales (un responsable y tres redactores), en La sexta sólo hay una persona que se encarga de este tema, Ángela Bonachera. Ambas entrevistadas descartan la retransmisión en directo a través de vídeo porque, tal y como afirma Bonachera "ya lo hacemos en la tele". La redactora jefa de RTVE.es considera Twitter como "la red natural para compartir información política y de última hora" destacando el formato del vídeo como el que mejor funciona en esta red social. Para Estefanía de Antonio

"el vídeo es un elemento diferencial respecto a otros medios y la marca más reconocible de RTVE. Tenemos más señales de televisión que ningún otro medio, por ello la capacidad de poder dar en directo la reacción de los políticos desde casi cualquier provincia de España fue un valor a explotar". 


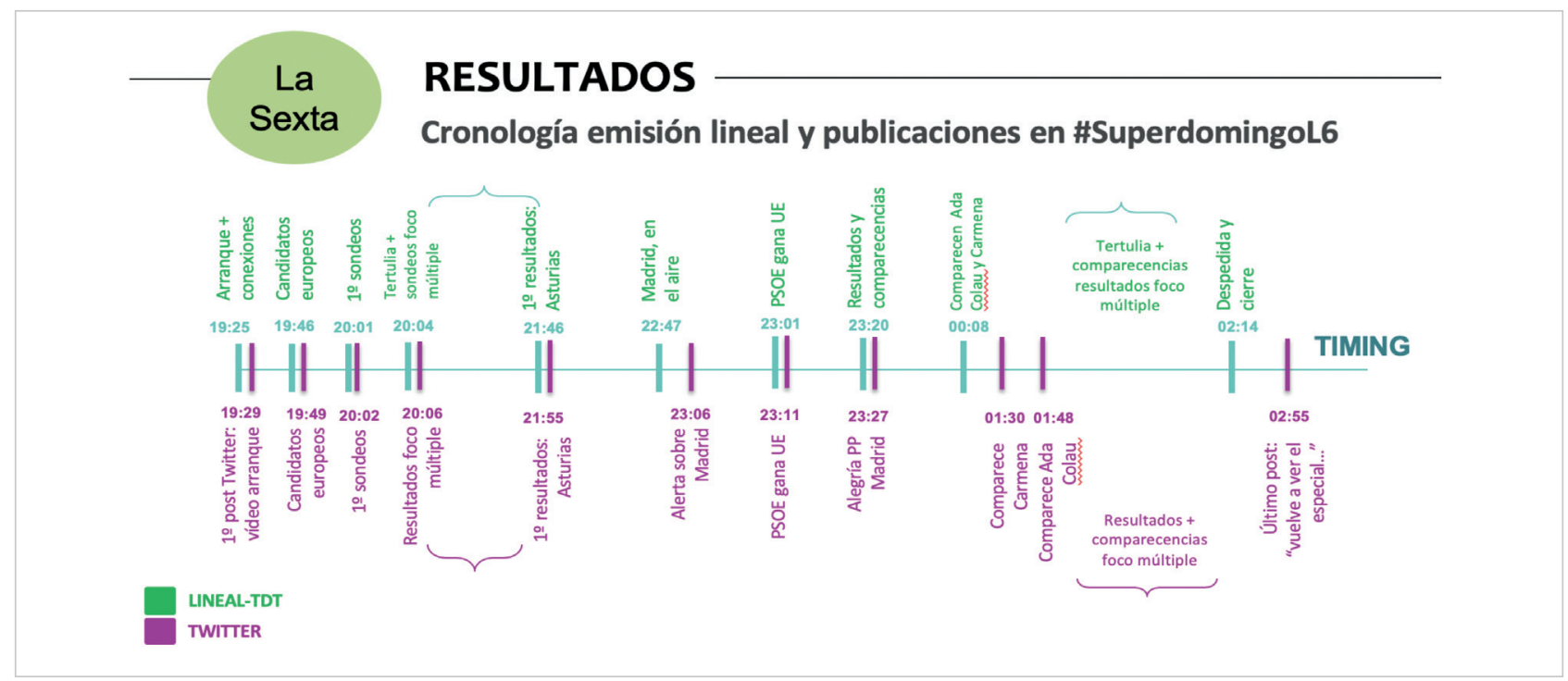

Imagen 2. Línea de tiempo: emisión lineal y publicaciones en RRSS en La sexta

Así se refleja en el análisis realizado, en el que 170 publicaciones de Twitter incorporaban vídeos o gifs. Los más reproducidos fueron las despedidas de Manuela Carmena en Madrid y de Ada Colau en Barcelona:

https://twitter.com/24h_tve/status/1132776132065972224

https://twitter.com/rtve/status/1132777643869294592

https://twitter.com/24h_tve/status/1132775295692361728

La actividad de las cadenas de TV en Twitter comenzó de forma casi simultánea a la emisión lineal: La sexta fue más rápida en sincronizar las dos actividades, ya que a los 4 minutos de empezar en directo lanzó el primer tweet con el hashtag \#SuperdomingoL6, mientras que la diferencia entre el arranque del programa especial en TV lineal y la primera publicación en \#26MTúDecides fue de 9 minutos. Comparativamente, la oferta de contenidos informativos por ambas cadenas en sus múltiples canales (TDT y RRSS), en cuanto a la frecuencia de las publicaciones, las temáticas y los focos informativos, discurrió de forma bastante paralela, aunque las RRSS fueron por detrás de la emisión lineal difundiendo los resultados.

\subsection{La conversación social en Twitter: live-tweeting de \#SuperDomingoL6 y \#26MTúDecides}

El debate que los usuarios generaron en Twitter alrededor de los hashtags propuestos durante la emisión de los especiales informativos de La sexta y La 1 muestra diferencias sustanciales entre ambas cadenas. Como primer dato señalamos que sólo la conversación en torno a la emisión especial de La sexta logra posicionarse entre los espacios televisivos más comentados de la semana. Según datos de Kantar Media-KSTR, ocupó el cuarto lugar con 35,3K tweets entre las 23:33h y las 02:14h.

El volumen de publicaciones recuperadas bajo el hashtag \#SuperDomingoL6 revela una mayor actividad por parte de los usuarios que tweetearon en directo el especial de Al rojo vivo, que consiguió alzarse también con el mejor dato de

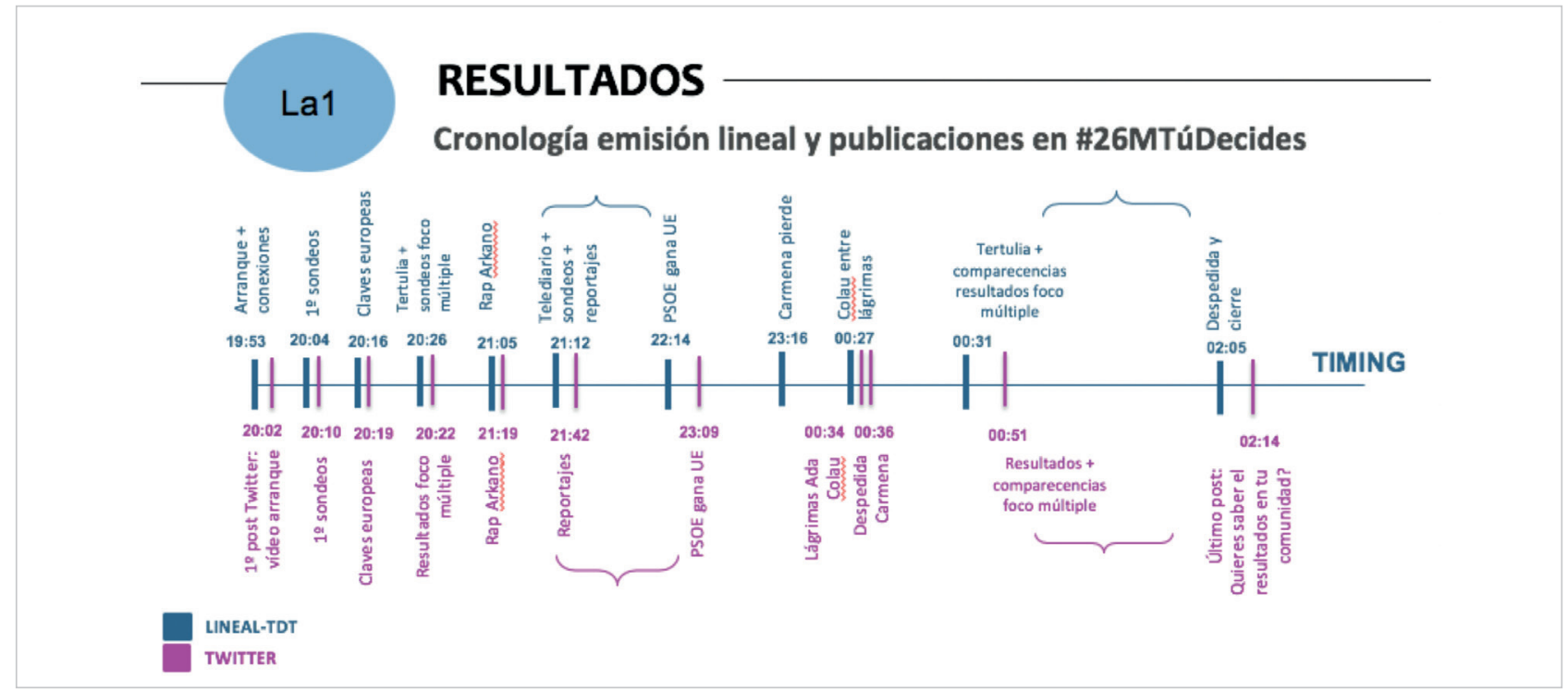

Imagen 3. Línea de tiempo: emisión lineal y publicaciones en RRSS en La 1 
audiencia lineal. Los retweets fueron el tipo de mensaje más popular en los dos programas, reflejando una tendencia de los usuarios a compartir o redistribuir las publicaciones realizadas por otros.

La conversación social mantuvo una línea ascendente desde el inicio de ambos programas y hasta casi la una de la madrugada. Sin embargo, tanto en el especial de Al rojo vivo como en el caso del 26M: Tú decides se observan fluctuaciones que ayudan a determinar cómo se comportaron los usuarios a medida que las cadenas avanzaban los resultados del recuento en el número de votos. Su mayor o menor implicación en la discusión generada en Twitter en torno a esas informaciones también queda

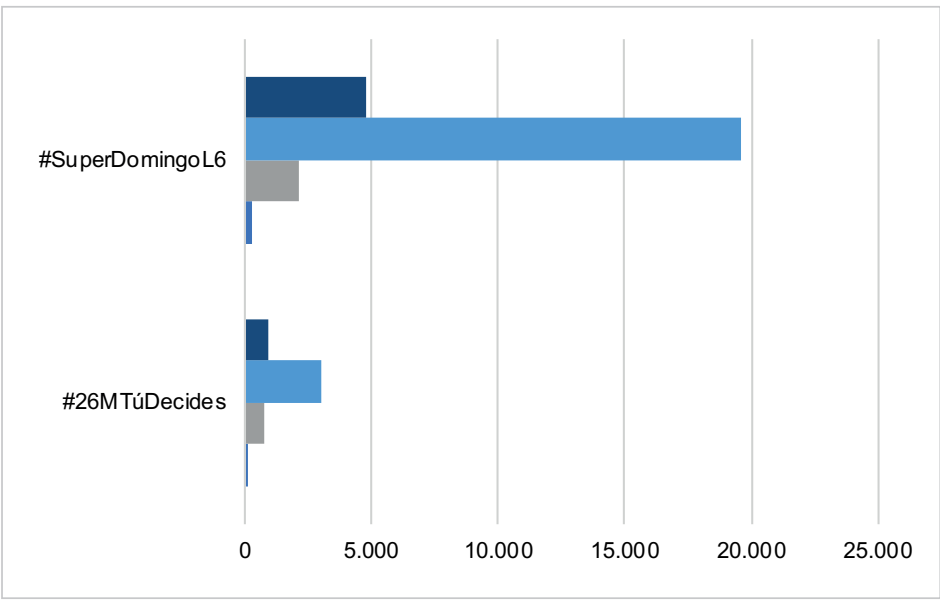

Gráfico 1. Volumen y tipo de mensajes publicados en Twitter con los hashtags \#SuperDomingoL6 y \#26MTúDecides patente en los altibajos que se detectan en la actividad de los live-tweeters (Pedersen et al., 2015).

El momento más comentado y uno de los más retweeteados por la audiencia social de los dos programas objeto de análisis se produjo a medianoche, coincidiendo con el instante en el que se dio a conocer que Manuela Carmena perdía la alcaldía de Madrid. Los minutos posteriores acumularon, igualmente, un alto volumen de publicaciones, llegando a alcanzar el máximo número de retweets entre las 00:30h y las 0:44h.

La interacción que se produjo entre los usuarios que comentaron en directo los especiales informativos de la noche electoral del 26 de mayo determina qué perfiles alcanzaron una mayor popularidad dentro de la red, así como las comunidades de usuarios que se crearon en torno a ellos. Los tweets de Pablo Villalba (@pablocoptero) y los de la periodista Marta Flich (@martaflich) fueron los que tuvieron una mayor difusión en Twitter bajo el hashtag \#SuperDomingoL6. El mensaje de la periodista sobre el momento en el que desde la sede del PP se realizaron gestos soeces mientras comparecía Manuela Carmena consiguió 1.757 retweets, siendo la publicación más compartida por los usuarios.

https://twitter.com/martaflich/status/1132776179759484929

Los tweets de Simon Derek (@simonderek) y los del guionista y director Guillem Clua (@guillemclua) fueron los que más se propagaron con el hashtag oficial del programa de La 1. En ambos casos, estuvieron dirigidos a lamentar la pérdida de Carmena como alcaldesa de Madrid.

Las comunidades que se crearon alrededor de estos perfiles señalan la afinidad de los usuarios con respecto a la postura ideológica mostrada en los mensajes, ya que como ha quedado patente en investigaciones anteriores (Congosto; Aragón, 2012; Larsson; Moe, 2012), se tiende a retweetear aquello con lo que se está de acuerdo. El grafo generado para la edición de Al rojo vivo muestra un total de 258 comunidades, agrupadas en torno a los perfiles de tweeteros anónimos, periodistas y de las cuentas oficiales tanto del programa que emitió el especial como del canal de noticias de $L a$ sexta. Lo mismo sucede en el caso del 26M: Tú decides, en el que se detectan 148 comunidades, entre las que sobresalen las formadas alrededor de los dos usuarios más retweeteados y de los perfiles de RTVE, Televisión Española y 24 horas.

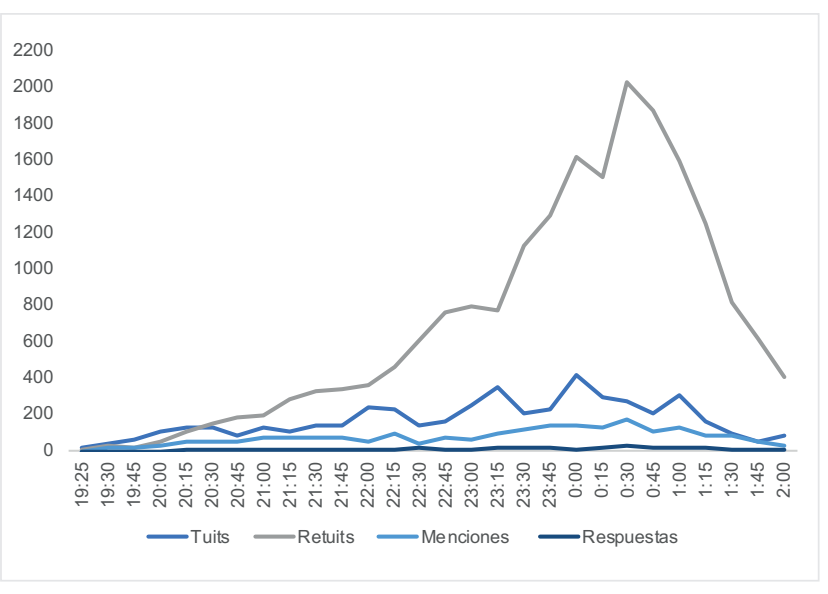

Hashtag \#SuperDomingoL6

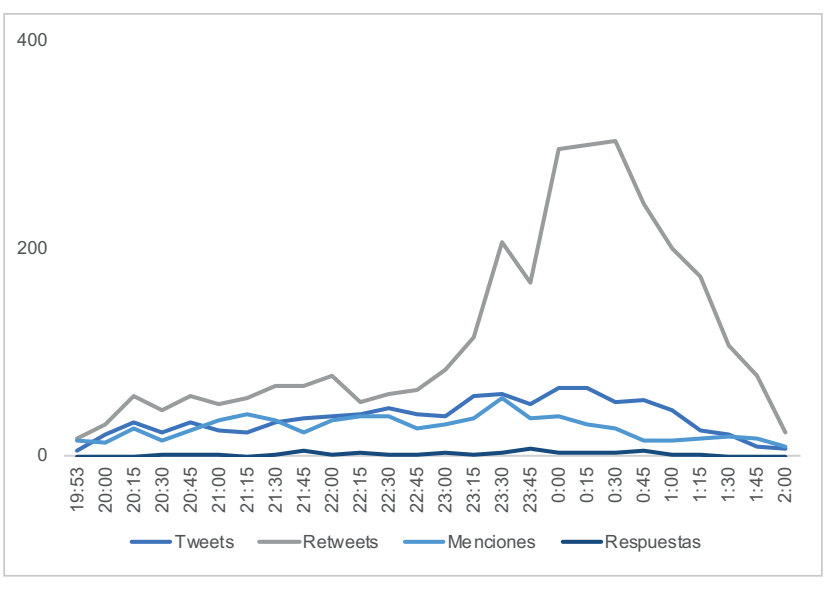

Hashtag \#26MTúDecides

Gráfico 2. Evolución de la conversación en Twitter 


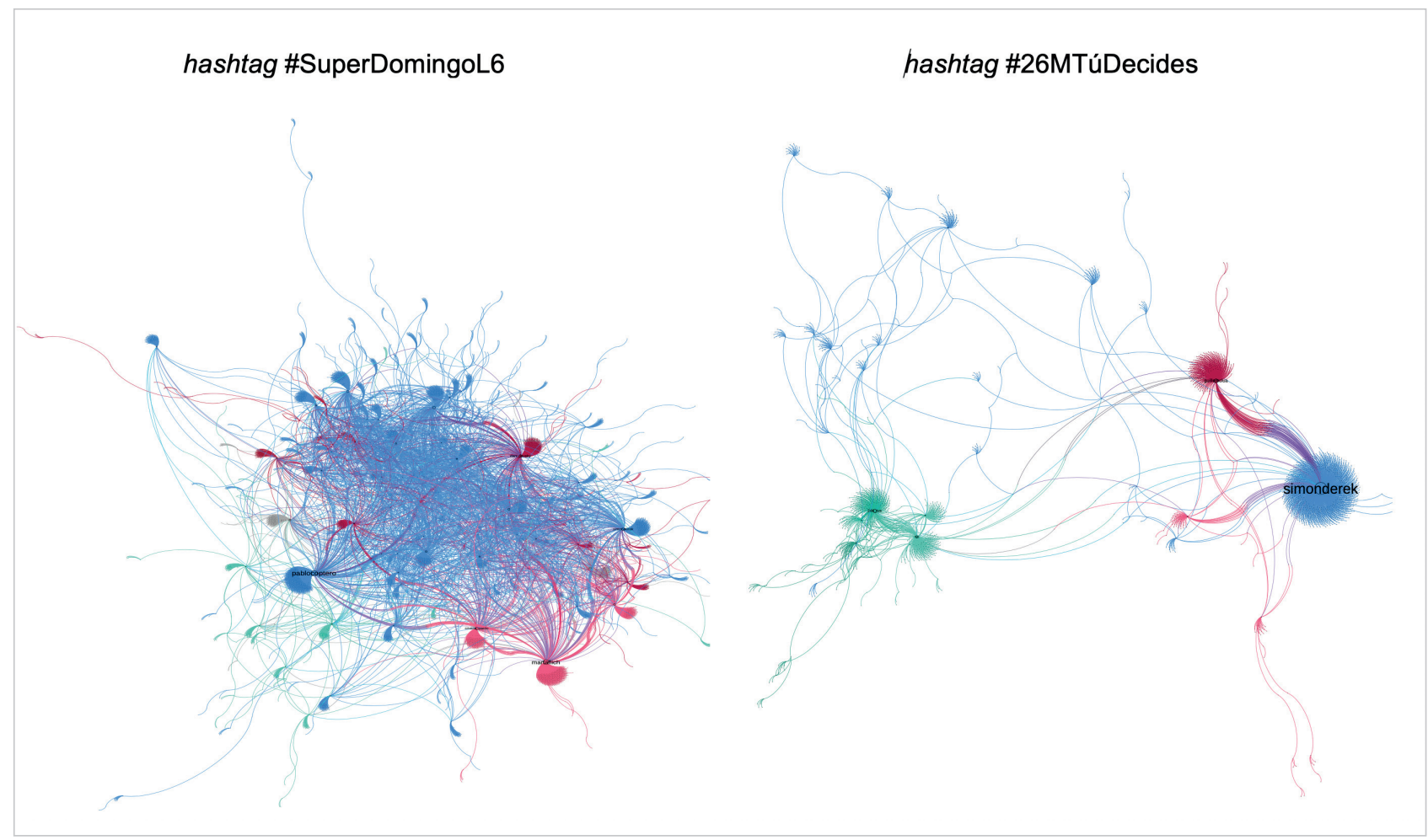

Gráfico 3. Red de retweets.

Grafo generado empleando la distribución Force Atlas 2. Los colores indican la pertenencia de los usuarios a una determinada comunidad, distinguiendo entre tweeteros (cyan), periodistas (magenta) y profesionales del medio (burdeos), perfiles de la cadena o programa (verde), medios de comunicación (amarillo) y otras cuentas (gris).

En lo referente a las menciones, mecanismo que ayuda a evaluar quién estuvo en el foco de la conversación en Twitter (Hawthorne; Houston; McKinney, 2013), se observa que los usuarios tienden a nombrar en sus tweets a los candidatos políticos y sus partidos, así como a los perfiles de los medios de comunicación. Pablo Iglesias y Manuela Carmena fueron los dos candidatos más mencionados por parte de la audiencia que comentó Al rojo vivo: edición especial. Objetivo superdomingo con el hashtag oficial del programa, consiguiendo ser nombrados 371 y 254 veces respectivamente. El perfil de La sexta destaca también en el grafo al acumular 232 menciones. Los usuarios que comentaron en directo con el hashtag propuesto por el 26M: Tú decides optaron por remarcar en la conversación los perfiles de la corporación y de la cadena que emitía el programa. Manuela Carmena fue la candidata que acumuló un número más alto de menciones, mientras que el rapero Arkano (@SmoothArkano), que estuvo presente durante la emisión del especial de La 1 y realizó una improvisación musical, tuvo también una posición destacada en el diálogo.

Tabla 1. Perfiles más mencionados por los usuarios que comentaron con los hashtags \#SuperDomingoL6 y 26MTúDecides

\begin{tabular}{|l|l|l|l|l|l|}
\hline \multicolumn{1}{|c|}{ \#SuperDomingoL6 } & \multicolumn{1}{c|}{ 26MTúDecides } \\
\hline Usuario & \multicolumn{1}{|c|}{ Perfil } & Menciones & Usuario & Perfil \\
\hline Pablo Iglesias & @Pablo-Iglesias & 371 & RTVE & @rtve \\
\hline Manuela Carmena & @ManuelaCarmena & 254 & La 1 & @La1_tve \\
\hline La sexta & @laSextaTV & 232 & Manuela Carmena & @ManuelaCarmena \\
\hline Podemos & @ahorapodemos & 174 & Arkano & @SmoothArkano \\
\hline Al rojo vivo & @DebatAIRojoVivo & 154 & Carlos Franganillo & @cfranganillo \\
\hline
\end{tabular}

La cuenta de RTVE agrupó, así mismo, a la mayor de las 54 comunidades formadas entre los que tweetearon con el hashtag \#26MTúDecides, mencionando en sus publicaciones los perfiles de otros usuarios. En el grafo sobresalen, igualmente, la creada en torno al Partido Socialista y al presentador de Televisión Española Carlos Franganillo. En el caso de La sexta, se detectan 95 comunidades, aunque las tres de mayor relevancia están constituidas alrededor de los partidos políticos y sus principales candidatos. Manuela Carmena encabeza la comunidad que tiene un mayor peso, seguida de la creada por los usuarios que mencionaron en sus tweets a los líderes y partidos políticos de derecha. En tercer lugar, alejada de ésta, pero próxima a la de Carmena, se sitúa la comunidad formada en torno a Pablo Iglesias, lo que indica la existencia de vínculos entre ambas. 


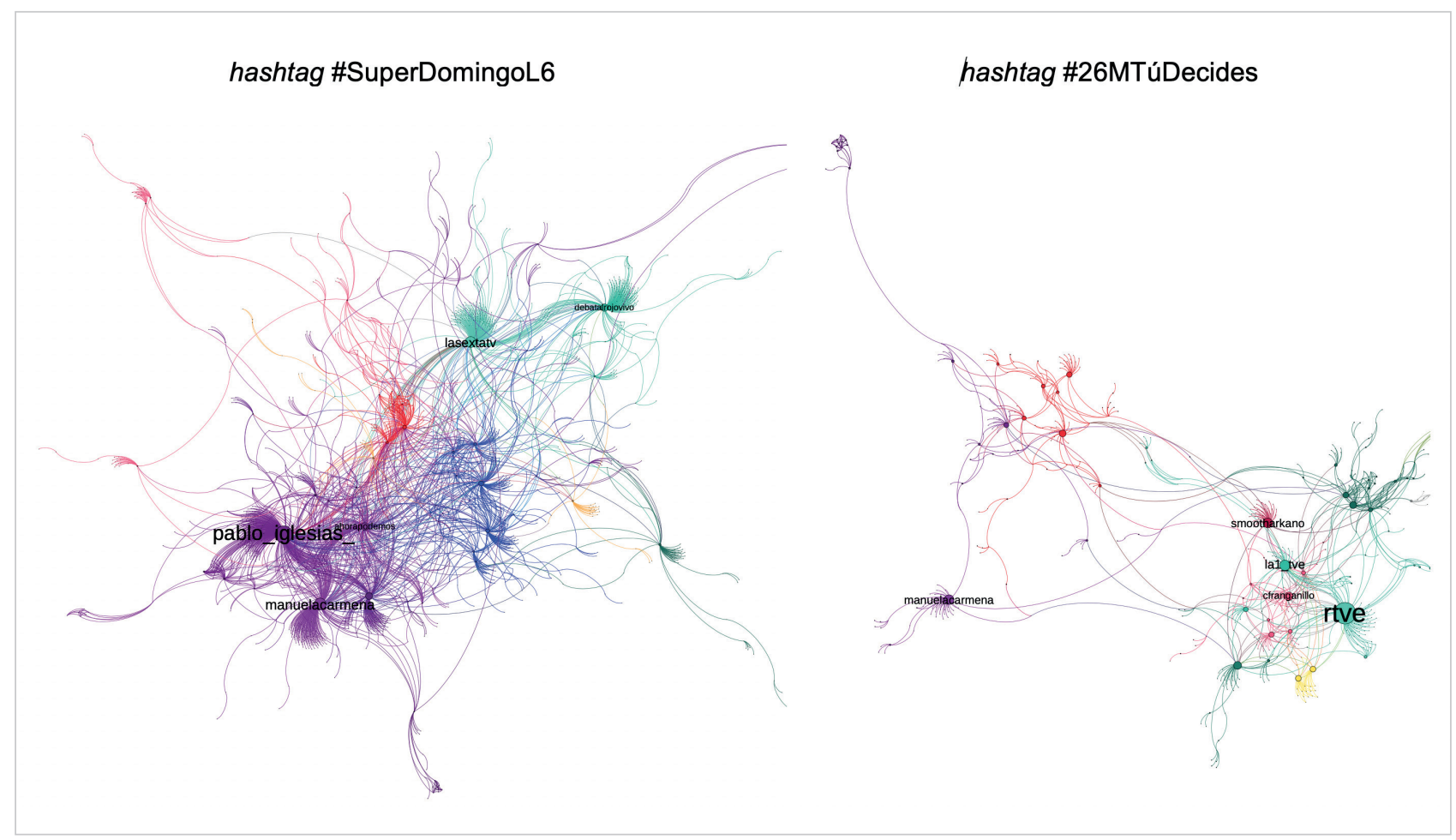

Gráfico 4. Red de menciones.

Grafo generado empleando la distribución Force Atlas 2. Los colores indican la pertenencia de los usuarios a una determinada comunidad, distinguiendo entre periodistas (magenta) y profesionales del medio (burdeos), perfiles de la cadena o programa (verde), medios de comunicación (amarillo) y otras cuentas (gris). Para los candidatos políticos y sus partidos, se emplean los siguientes códigos de colores: PSOE (rojo), PP (azul), Podemos, IU y Más Madrid (violeta) y Ciudadanos (naranja).

\section{Discusión y conclusiones}

Durante la noche electoral analizada las cadenas de televisión rompen las convenciones de la televisión lineal para el tratamiento de los eventos políticos y avanzan en la búsqueda de la audiencia social. Si bien los contenidos y focos informativos fueron similares en las emisiones lineales y RRSS, éstas fueron por detrás de la emisión en directo de la TV tradicional a la hora de dar resultados. También se detectaron diferencias en las dos cadenas analizadas. Se registran más medios y variedad de contenidos en la emisión lineal en La 1 (escenografía, reportajes variados, cobertura de las elecciones europeas...) que, sin embargo, la audiencia no respalda con el dato de audimetría ni con sus comentarios en las redes sociales.

El despliegue de un mayor número de recursos visuales y de contenido en $L a 1$ pudo ralentizar el ritmo informativo y la actualización casi inmediata buscada por La sexta, que ha primado el valor informativo de la actualización frente a la contextualización y profundización en el mensaje. De este modo, mientras que la La sexta puso la atención únicamente en los datos y el análisis, La 1 amplió su enfoque con reportajes de contextualización y otras piezas de divulgación de aspectos complementarios a la jornada electoral. Esto se tradujo en una mayor dispersión y la pérdida de la actualidad durante varios minutos en los que La sexta mantuvo el pulso a la última hora. Además, la incorporación del Telediario al programa especial informativo supuso una disrupción más en el ritmo narrativo, ya que obligó a La 1 a recapitular contenidos frente al avance de La sexta hacia los resultados más inmediatos. En este contexto resultó especialmente desafortunada la intervención de Ana Blanco en La 1 alrededor de las 22:50h en la que aún se refirió a los datos del sondeo que daban una posible victoria a la izquierda en Madrid, cuando ya algunos colaboradores del canal público (y especialmente en el competidor privado) hablaban de la pérdida de la alcaldía de Madrid por Manuela Carmena.

La estrategia de ambos programas en las RRSS es similar en cantidad de publicaciones, frecuencias, contenidos y focos informativos. Coincide en el mayor esfuerzo de los canales en torno a Twitter, pero difiere en aspectos clave para el engagement con el público como el diseño de los posts (mucho vídeo en La 1 frente al predominio de imagen fija en La sexta) o la visibilidad en las distintas redes (nula presencia de La sexta en YouTube frente a la retransmisión en simultáneo de La 1). Esta circunstancia hay que relacionarla, por otra parte, con la composición del equipo que gestiona las redes sociales en ambas cadenas: cuatro profesionales en TVE frente a una sola persona en La sexta.

La 1 se esfuerza en alcanzar a un público potencialmente más joven y ávido del uso de las RRSS, especialmente las más audiovisuales como Instagram y YouTube.

La audiencia, por su parte, se centra más durante esta noche en compartir y en redistribuir publicaciones realizadas por otros usuarios. El volumen de tweets recuperados bajo el hashtag \#SuperDomingoL6 revela una mayor actividad por 
parte de los usuarios que tweetearon en directo el especial de Al rojo vivo, que consiguió alzarse también con el mejor dato de audiencia lineal. Por otro lado, las comunidades que se crearon alrededor de estos perfiles señalan la afinidad de los usuarios con respecto a la postura ideológica mostrada en los mensajes. Se constata que se tiende a retweetear aquello con lo que se está de acuerdo.

Por lo tanto, el evento político se confirma como un momento relevante en el que las cadenas de televisión aplican innovaciones y una comunicación híbrida para relacionarse con la audiencia activa no sólo en la interpretación de los mensajes periodísticos, sino también en la producción y el debate de contenidos. Los resultados de las estrategias de las cadenas televisivas no son lineales y están influidos por diferentes factores, entre ellos la predisposición de la audiencia, que conviene analizar en próximas investigaciones.

\section{Referencias}

Abitbol, Alan; Lee, Sun-Young (2017). "Messages on CSR-dedicated Facebook pages: What works and what doesn't". Public relations review, v. 43, n. 4, pp. 796-808.

https://doi.org/10.1016/j.pubrev.2017.05.002

Alonso-González, Marián (2018). "Infoentretenimiento y política: el caso de las elecciones de 2015 y 2016 en España”. Revista de comunicación de la Seeci, n. 44, pp. 87-113. https://doi.org/10.15198/seeci.2017.44.87-113

Azurmendi, Ana (2018): “Reconectar con la audiencia joven. Narrativa transmedia para la transformación de la televisión de servicio público en España, Francia, Alemania y Reino Unido”. Revista latina de comunicación social, n. 73, pp. 927-944.

https://doi.org/10.4185/RLCS-2018-1289

Blondel, Vincent D.; Guillaume, Jean-Loup; Lambiotte, Renaud; Lefebvre, Etienne (2008). "Fast unfolding of communities in large networks". Journal of statistical mechanics: Theory and experiment, v. 2008, n. 10. https://doi.org/10.1088/1742-5468/2008/10/P10008

Bruns, Axel; Burgess, Jean E. (2011). "The use of Twitter hashtags in the formation of ad hoc publics". In: Proceedings of the $6^{\text {th }}$ European Consortium for Political Research (ECPR) general conference. University of Iceland, Reykjavik. https://eprints.qut.edu.au/46515

Burnet, Simon; Bloice, Lyndsay (2016). "Linking for influence: Twitter linked content in the Scottish referendum televised debates". Journal of information science, v. 42, n. 3, pp. 396-409.

https://doi.org/10.1177/0165551515624355

Comscore (2019). Beyond the headlines. Unpacking common narratives in media and advertising.

https://www.comscore.com/Insights/Presentations-and-Whitepapers/2019/Beyond-the-Headlines-UnpackingCommon-Narratives-in-Media-and-Advertising

Cha, Jiyoung (2016). "Television use in the $21^{\text {st }}$ century: An exploration of television and social television use in a multiplatform enviroment". First Monday, v. 21, n. 2.

https://doi.org/10.5210/fm.v21i2.6112

Chadwick, Andrew (2013). The hybrid media system: Politics and power. Oxford: Oxford University Press. ISBN: 9780 199759484

Congosto, María-Luz; Aragón, Pablo (2012). "Twitter, del sondeo a la sonda: nuevos canales de opinión, nuevos métodos de análisis". Más poder local, n. 12, pp. 50-56.

Dodd, Melissa; Collins, Steve (2017). "Public relations message strategies and public diplomacy 2.0: An empirical analysis using Central-Eastern European and Western Embassy Twitter accounts". Public relations review, v. 43, n. 2, pp. 417425.

https://doi.org/10.1016/j.pubrev.2017.02.004

Gallego, Francisco (2013): "Social TV analytics: Nuevas métricas para una nueva forma de ver televisión". Index.comunicación: Revista científica de comunicación aplicada, v. 3, n. 1, pp. 13-39.

http://journals.sfu.ca/indexcomunicacion/index.php/indexcomunicacion/article/view/49

Gálvez-Rodríguez, María-del-Mar; Caba-Pérez, Carmen; López-Godoy, Manuel (2014). “Facebook: A new communication strategy for non-profit organisations in Colombia". Public relations review, v. 40, n. 5, pp. 868-870.

https://doi.org/10.1016/j.pubrev.2014.10.002

Gálvez-Rodríguez, María-del-Mar; Sáez-Martín, Alejandro; García-Tabuyo, Manuela; Caba-Pérez, Carmen (2018). “Exploring dialogic strategies in social media for fostering citizens' interactions with Latin American local governments". Public relations review, v. 44, n. 2, pp. 265-276.

https://doi.org/10.1016/j.pubrev.2018.03.003 
García-Orosa, Berta; López-García, Xosé (2019). “Language in social networks as a communication strategy: Public administration, political parties and civil society". Communication \& society, v. 32, n. 1.

https://doi.org/10.15581/003.32.1.107-125

Grčić, Klara; Babac, Marina-Bagić; Podobnik, Vedran (2017). “Generating politician profiles based on content analysis of social network datasets". Journal of universal computer science, v. 23, n. 3, p. 236-255.

https://doi.org/10.3217/jucs-023-03-0236

Harrington, Stephen; Highfield, Tim; Bruns, Axel (2013). "More than a backchannel: Twitter and television". Participations. Journal of audience \& reception studies, v. 10, n. 1.

http://snurb.info/files/2014/More\%20than\%20a\%20Backchannel.pdf

Hawthorne, Joshua; Houston, J. Brian; McKinney, Mitchell S. (2013). "Live-tweeting a presidential primary debate: Exploring new political conversations". Social science computer review, v. 31, n. 5, pp. 552-562.

https://doi.org/10.1177/0894439313490643

Haythornthwaite, Caroline (1996). "Social network analysis: An approach and technique for the study of information exchange". Library \& information science research, v. 18, n. 4, pp. 323-342.

https://doi.org/10.1016/S0740-8188(96)90003-1

Himelboim, Itai; Smith, Marc A.; Rainie, Lee; Shneiderman, Ben; Espina, Camila (2017). "Classifying Twitter topic-networks using social network analysis". Social media + society, v. 3, n. 1, pp. 1-13.

https://doi.org/10.1177/2056305117691545

Ji, Yi-Grace; Chen, Zifei-Fay; Tao, Weiting; Li, Zongchao-Cathy (2018). "Functional and emotional traits of corporate social media message strategies: Behavioral insights from S\&P 500 Facebook data". Public relations review, v. 45, n. 1, pp. 88-103.

https://doi.org/10.1016/j.pubrev.2018.12.001

Jungherr, Andreas (2016). "Twitter use in election campaigns: A systematic literature review". Journal of information technology \& politics, v. 13, n. 1, pp. 72-91.

https://doi.org/10.1080/19331681.2015.1132401

Jürgens, Pascal; Jungherr, Andreas (2015). "The use of Twitter during the 2009 German national election". German politics, v. 24, n. 4, pp. 469-490.

https://doi.org/10.1080/09644008.2015.1116522

Larsson, Anders-Olof; Moe, Hallvard (2012). "Studying political microblogging: Twitter users in the 2010 Swedish election campaign". New media \& society, v. 14, n. 5, pp. 729-747.

https://doi.org/10.1177/1461444811422894

Lin, Yu-Ru; Keegan, Brian; Margolin, Drew; Lazer, David (2014). “Rising tides or rising stars?: Dynamics of shared attention on Twitter during media events". PLoS one, v. 9, n. 5, e94093.

https://doi.org/10.1371/journal.pone.0094093

Liu, Wenlin; Lai, Chih-Hui; Xu, Weiai Wayne (2018). "Tweeting about emergency: A semantic network analysis of government organizations' social media messaging during hurricane Harvey". Public relations review, v. 44, n. 5, pp. 807-819. https://doi.org/10.1016/j.pubrev.2018.10.009

Livingstone, Sonia; Lunt, Peter (1994). “The mass media, democracy and the public sphere". In: Livingstone, Sonia; Lunt, Peter. Talk on television: Audience participation and public debate. Londres: Routledge, pp. 9-35. ISBN: 9780415077385 http://www.Ise.ac.uk/media@Ise/WhosWho/AcademicStaff/SoniaLivingstone/pdf/Talk-on-TV-ch-2-for-eprint.pdf

López-Rabadán, Pablo; Mellado, Claudia (2019). "Twitter as a space for interaction in political journalism. Dynamics, consequences and proposal of interactivity scale for social media". Communication \& society, v. 32, n. 1, pp. 1-18. https://doi.org/10.15581/003.32.1.1-18

Masip, Pere; Ruiz-Caballero, Carlos; Suau, Jaume (2019). "Active audiences and social discussion on the digital public sphere. Review article". El profesional de la información, v. 28, n. 2, e280204.

https://doi.org/10.3145/epi.2019.mar.04

Orihuela, José-Luis (2011). Mundo Twitter. Barcelona: Alienta. ISBN: 9788492414895

Ott, Larissa; Theunissen, Petra (2015). "Reputations at risk: Engagement during social media crises". Public relations review, v. 41, n. 1, pp. 97-102.

https://doi.org/10.1016/j.pubrev.2014.10.015

Painter, David-Lynn (2015). “Online political public relations and trust: Source and interactivity effects in the 2012 US presidential campaign". Public relations review, v. 41, n. 5, pp. 801-808.

https://doi.org/10.1016/j.pubrev.2015.06.012 
Pedersen, Sarah; Baxter, Graeme; Burnett, Simon; Goker, Ayse; Corney, David; Martin, Carlos (2015). "Backchannel chat: Peaks and troughs in a Twitter response to three televised debates during the 2014 Scottish independence referendum campaign". In: Parycek, P.; Edelmann, N. (eds.). Proceedings of the 2015 International conference for e-democracy and open government (CeDEM15), 20-22 May Krems, Austria, pp. 105-117.

https://rgu-repository.worktribe.com/output/246360

Saavedra-Llamas, Marta; Rodríguez-Fernández, Leticia; Barón-Dulce, Gema (2016). “Audiencia social en España: Estrategias de éxito en la televisión nacional". Icono 14, v. 13, n. 2.

https://doi.org/10.7195/ri14.v13i2.822

Shah, Dhavan V.; Culver, Kathleen-Bartzen; Hanna, Alexander; Macafee, Timothy; Yang, JungHwan (2015). “Computational approaches to online political expression: Rediscovering a 'science of the social'”. In: Coleman, Stephen; Freelon, Deen (eds.). Handbook of digital politics. Northampton, MA: Edward Elgar, pp. 281-305. ISBN: 9781782548751

Shamma, David A.; Kennedy, Lyndon; Churchill, Elizabeth F. (2009). "Tweet the debates: understanding community annotation of uncollected sources". In: Proceedings of the first Sigmm Workshop on social media.

https://doi.org/10.1145/1631144.1631148

Spyridou, Lia-Paschalia (2018). "Analyzing the active audience: Reluctant, reactive, fearful, or lazy? Forms and motives of participation in mainstream journalism". Journalism, v. 20, n. 6, pp. 827-847.

https://doi.org/10.1177/1464884918784733

Taylor, Maureen; Kent, Michael L. (2010). "Anticipatory socialization in the use of social media in public relations: A content analysis of PRSA's public relations tactics". Public relations review, v. 36, n. 3, pp. 207-214.

https://doi.org/10.1016/j.pubrev.2010.04.012

Utz, Sonja; Schultz, Friederike; Glocka, Sandra (2013). “Crisis communication online: How medium, crisis type and emotions affected public reactions in the Fukushima Daiichi nuclear disaster". Public relations review, v. 39, n. 1, pp. 40-46. https://doi.org/10.1016/j.pubrev.2012.09.010

Vesnic-Alujevic, Lucia (2012). "Political participation and web 2.0 in Europe: A case study of Facebook". Public relations review, v. 38, n. 3, pp. 466-470.

https://doi.org/10.1016/j.pubrev.2012.01.010

Wasserman, Stanley; Faust, Katherine (2013). Análisis de redes sociales: métodos y aplicaciones. Madrid: CIS. ISBN: 978 8474766318

Waters, Richard D.; Jamal, Jia Y. (2011). “Tweet, tweet, tweet: A content analysis of nonprofit organizations' Twitter updates". Public relations review, v. 37, n. 3, pp. 321-324.

https://doi.org/10.1016/j.pubrev.2011.03.002

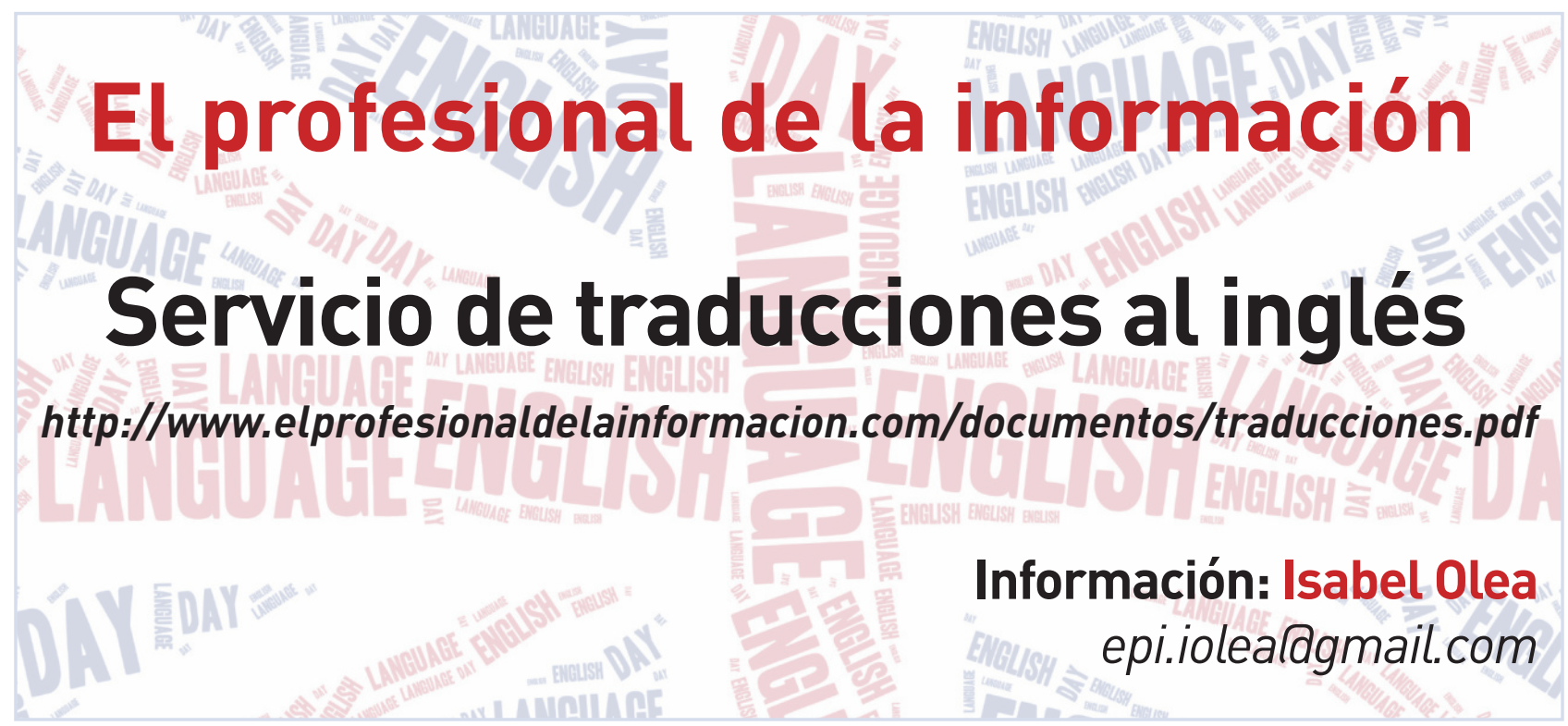

